17. Гусева М.А., Хмелевская А.Г., Гусев И.Д. Съемная накладка на типовой манекен для имитации формы поверхности тела индивидуальной фигуры человека. Патент на полезную модель RU 156812 U1. от 21.05.2015. Опубл. 20.11.2015 Бюл. № 32.

18. Петросова И.А., Андреева Е.Г. Разработка технологии трехмерного сканирования для проектирования виртуальных манекенов фигуры человека и 3D моделей одежды. - Москва, 2015.

19. Гусева М.А., Андреева Е.Г., Петросова И.А. Способ проектирования конструкций одежды на основе совмещения виртуальных образов типовой и индивидуальной фигур. Заявка № 2017111453 от 05.04.2017.

20. Андреева Е.Г., Петросова И.А., Гусева М.А., Шанцева О.А. Анализ существующих способов проведения виртуальной примерки. // В сборнике: Материалы докладов 50-й международной научно-технической конференции преподавателей и студентов, посвященной году науки 50-я международная научно-техническая конференция: в 2-х томах. 2017. С. 142-145.

21. Гетманцева В.В., Гончарова А.С., Никитина Н.Г., Андреева Е.Г. Влияние показателей физикомеханических свойств тканей на пространственную форму плечевого изделия// Известия вузов. Технология текстильной промышленности. - 2011, № 6. С.88-94.

\title{
Рябова А.А. \\ Внедрение элементов искусственного интеллекта в информационные системы управления
}

ФГБОУ ВПО «РЭУ им. Г.В. Плеханова» филиал в г. Пятигорске

(Россия, Пятигорск)

doi:10.18411/spc-16-01-2018-10

idsp: 000001:spc-16-01-2018-10

\begin{tabular}{|c|c|}
\hline $\begin{array}{l}\text { Аннотация } \\
\text { В статье исследованы вопросы разности понимания ис } \\
\text { стественного интеллекта в процессе принятии решения. }\end{array}$ & скусственного \\
\hline $\begin{array}{c}\text { Приведен пример внедрения элементов } \\
\text { нфкусственного } \\
\text { нформационную системы по выбору смартфона. }\end{array}$ & интеллекта \\
\hline
\end{tabular}

\footnotetext{
Abstract

In the article questions of difference of understanding of artificial and natural intelligence in the decision-making process are investigated.

An example of the introduction of artificial intelligence elements into the information system for choosing a smartphone is given.
}

Искусственный интеллект (ИИ) сегодня одно из важнейших направлений исследований ученых и инженеров. Причем рассматриваются как системы, созданные с его частичным использованием: например распознавание текстов, бытовые роботы, до возможности полной замены творческого труда человека искусственным. В настоящее время в самых различных областях науки и техники требуется автоматическое выполнение тех задач, которые раньше под силу были только человеку. Сегодня системы, как программные, так и аппаратные, созданные на основе искусственного интеллекта находят все большее применение в технике. Это и автопилотируемые автомобили с электроникой с использованием ИИ, и новейшие роботы, участвующие в производстве чего-либо, и компьютерные программы, которые включают ИИ в качестве советующих или систем поддержки принятия решений.

На современном этапе развития исследований в этой области до создания систем полного ИИ довольно далеко, поэтому нам приходится ограничиваться лишь частичным вмешательством ИИ в интеллектуальные системы. Это, прежде всего, программные средства. Например, экспертные системы, системы распознавания образов и др.

Среди многих точек зрения касательно вопроса понятия ИИ преобладают следующие три: 
1) фундаментальные исследования, в процессе которых разрабатываются новые модели и методы для решения задач, считающихся интеллектуальными и не поддававшихся ранее формализации и автоматизации.

2) исследования, связанные с новыми идеями решения задач на ЭВМ, с разработкой новых технологий программирования и переходом к компьютерам не фоннеймановской архитектуры.

3) исследования, в процессе которых появляется множество прикладных систем, способных решать задачи, для которых ранее создаваемые системы были не пригодны.

Чтобы облегчить понимание такого явления как искусственный интеллект, выявим его признаки на основе сравнения двух основных видов интеллекта естественного (интеллекта человека) и искусственного. Как известно, понятие интеллект отождествляют с понятием мышления. Если говорить об интеллекте человека, это утверждение верно, однако, в случае искусственного - не совсем.

Как естественный, так и искусственный интеллект использует различные типы информационных единиц - признаки, понятия или концепты, образы, модели и пр. При этом существует значительное различие в способах реализации и возможностях этих двух видов интеллекта.

Процесс восприятия информации у человека можно представить в виде цепочки следования: семантизация понятий $>$ понимание $>$ получение выводов.

В процессе восприятия и понимания информации у человека возникает представление о проблемной ситуации, т.е. ментальная модель, которая позже может быть преобразована в более формализованный вид.

Что касается искусственного интеллекта, то в процессе принятия решения интеллектуальной системой отсутствует этап понимания, а, следовательно, речь идет уже не об интеллекте, а о моделях реализации интеллекта. Это существенное различие и составляет грань между интеллектом человека и искусственным интеллектом.

Таким образом, искусственная интеллектуальная система, обладающая механизмами искусственного мышления, получает возможность упорядочивать и корректировать свои знания. Осуществляя повторный неоднократный ввод хранящейся в памяти информации, снова распознавая и сравнивая ее с содержимым памяти, тем самым выполняет неоднократный просмотр и коррекцию формируемых внутри образов (моделей внешнего мира) в непрерывном потоке информации. В действительности же, процесс осознания представляет собой ассоциативное воспоминание с обновлением и требует периодического распознавания информации, представляющей внутренне состояние и внешнюю среду.

Реализация подобных возможностей предполагает необходимость обеспечения следующих функций человеко-машинного интерфейса:

- поддержание диалога на уровне естественного языка с речевым и символьным вводом/выводом;

- ввод и адекватное восприятие естественно-языковых целеуказаний и команд оператора на выполнение требуемых прикладных задач;

- объяснение принимаемых решений на уровне естественного языка;

- естественно-языковое обучение понятиям и общим закономерностям, признакам объектов и ситуаций, правилам целесообразного поведения в типовых ситуациях.

Требования к функциям интеллектуальной системы определяются необходимостью достоверной идентификации текущего состояния среды для построения адекватной модели ситуации выбора и последующего принятия решений о рациональном порядке выполнения поставленной прикладной задачи. При этом очевидно, что эффективность функционирования интеллектуальной системы 
управления выбором будет во многом зависеть от заложенного набора ее возможностей, включая:

- сбор и выполнение предварительной обработки различных видов информации о текущем состоянии и воздействиях;

- распознавание образов;

- способность к оперативному обучению средств распознавания;

- способность отслеживать заданные типы объектов или изменений внешней среды с идентификацией их параметров.

Для подтверждения идей и теорий, изложенных выше, автором была поставлена задача разработки и реализации модели интеллектуальной системы по выбору смартфона. Работа выполнялась студентом 4 курса специальности «Информационные системы и технологии»ФГБОУ ВПО «РЭУ им. Г.В. Плеханова» филиал в г. Пятигорске Машином Алексеем под руководством автора.

Если не следить за рынком обновления смартфонов постоянно, то может быть затруднительно выбрать подходящий смартфон. Представленная интеллектуальная системас может дать ответы на самые важные вопросы по выбору смартфона. С еe помощью можно узнать, как выбрать смартфон, подходящий именно вам, какой смартфон лучше купить в 2017-2018 году, а также как сэкономить на покупке.

В процессе исследования предметной области были выявлены самые распространённые ошибки при выборе смартфона. Например, что если в смартфоне стало вдвое больше ядер процессора и оперативной памяти, увеличилось разрешение экрана и количество мегапикселей камеры, то он обязательно будет лучше. Это не совсем так. Результаты тестов смартфонов, после проверки устройств в лаборатории и при ежедневном использовании, наглядно показали, что многие параметры и характеристики не влияют на качество смартфона напрямую. Поэтому, если у вас смартфон прошлого или позапрошлого года, то совершенно необязательно менять его именно из-за «устаревших» характеристик.

Благодаря данной системе может быть подобран самый оптимальный вариант смартфона, исходя из запросов конкретного пользователя. Это поможет избежать переплаты за смартфон т.е. избежать покупки дорогого смартфона, если требуется смартфон ограниченного функционала.

При решении задачи был выбран метод ветвей и границ. Данный метод является одной из первых эффективных схем неявного перебора, идея которого состоит в том, что при решении задачи можно избежать полного перебора путем отбрасывания заведомо неоптимальных решений.

Идея метода состоит в следующем: решая задачу, необходимо разложить ее решение на множество возможных вариантов. В результате становится возможным отбрасывать решения целыми классами, если в них уже нет необходимости.

На рисунке 1 представлена функциональная модель системы.

Интерфейс приложения для реализации модели интеллектуальной системы представляет собой кнопочную систему управления. Работа в системе выглядит следующим образом:

1. Выбор одного из предложенных вариантов ответов.

2. После выбора остальные варианты отбрасываются.

3. Далее необходимо повторять первый пункт, пока не будет предложен оптимальный вариант смартфона.

В итоге подбирается самый подходящий смартфон на основе этих действий.

Для реализации этой идеи использовалась среда программирования Delphi, так как в алгоритме используются и алгебраические операции, и операции логики, необходимо использовать универсальный язык программирования, например: Pascal, Java, C++. 
В самом начале подбора смартфона следует выбрать по каким критериям будет производиться выбор. На рисунке 2 представлены критерии.

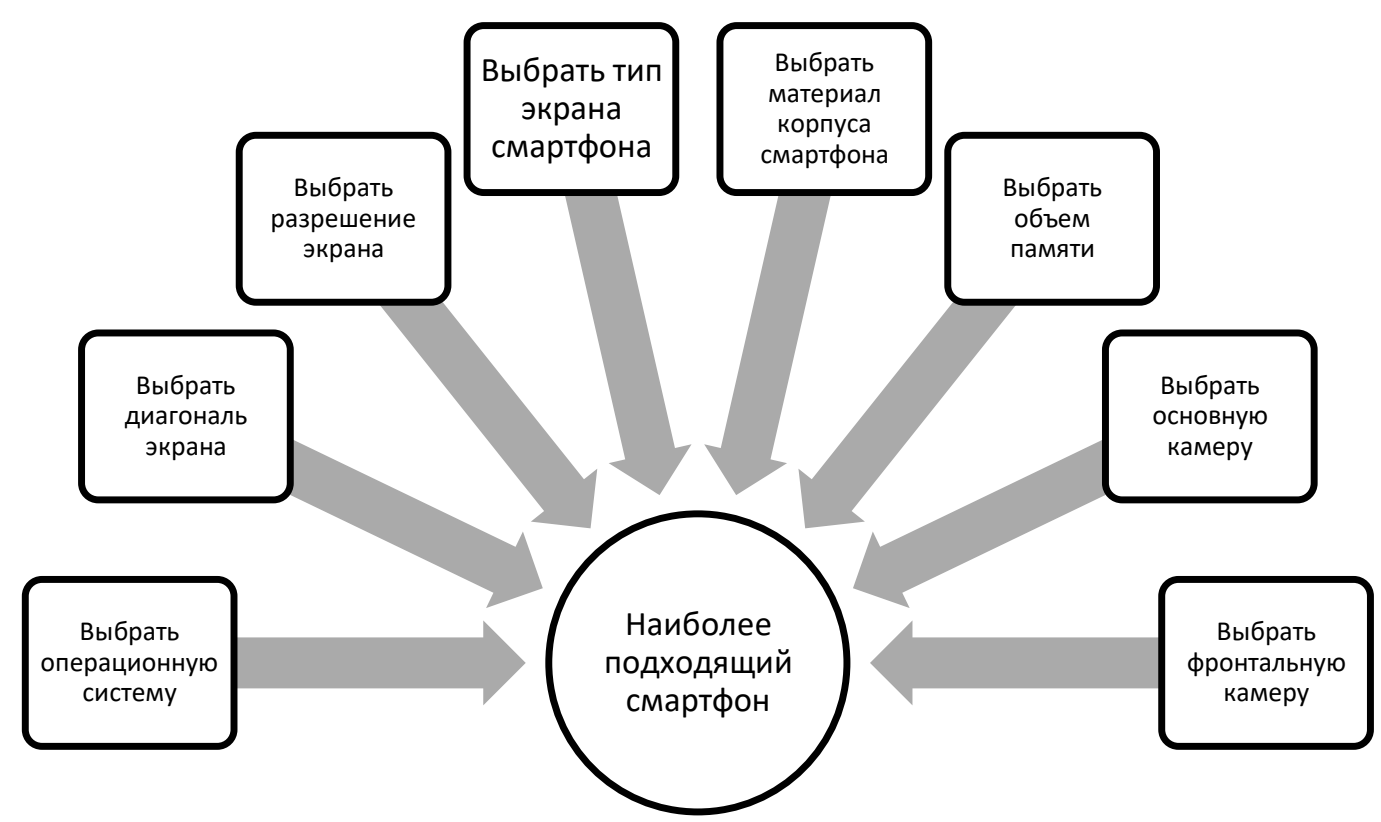

Рисунок 1 - Функциональная модель системь

\begin{tabular}{|c|c|c|c|}
\hline च Операционная система & च Тип экрана & $\square$ Материал корпуса & ■ Камера \\
\hline च Диагональ экрана & У Разрешение экрана & У Память смартфона & $\square$ Выбрать все \\
\hline
\end{tabular}

Рисунок2 -Выбор критериев отбора смартфона

В качестве примера рассмотрим критерий - операционная система (OC). Для ознакомления с особенностями данных ОС необходимо нажать на интересующее вас название, как показано на рисунке 3 После это в правой части формы отобразится краткая информация о выбранной ОС. Если пользователь не знает, что такое ОС, то система придет на помощь по нажатию на кнопку «Что такое ОС?»

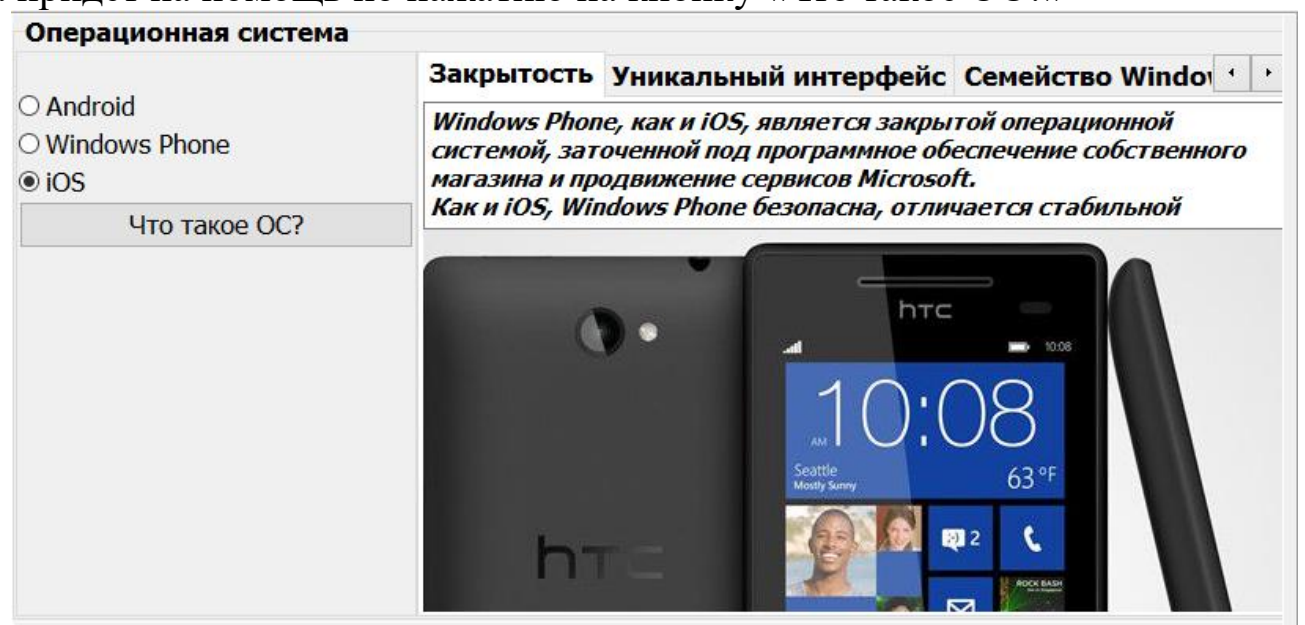

Рисунок3-Выбор операционной системы смартфона

Далее можно выбрать интересующую диагональ будущего устройства, как показано на рисунке 4. По нажатию на изображение необходимой диагонали смартфона отображается краткая информация о данной диагонали. 


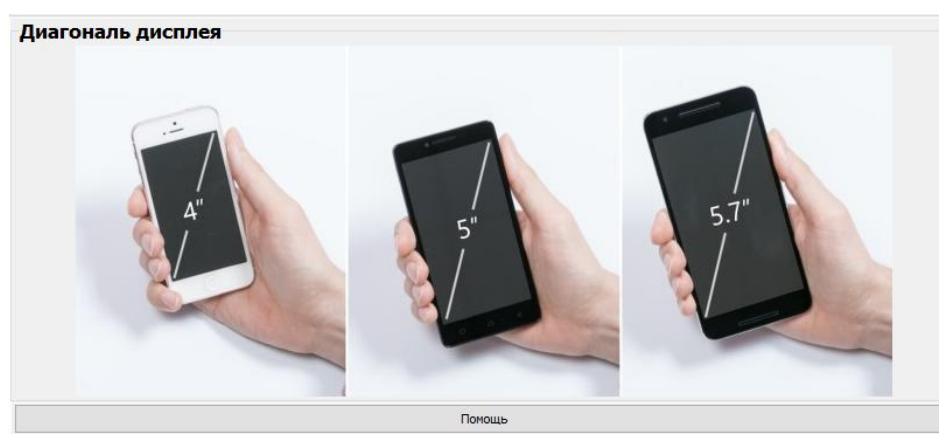

Рисунок4 -Выбор диагонали устройства

Для ознакомления с особенностями предпочитаемых данных материалов корпуса смартфона необходимо нажать на интересующее название, как показано на рисунке 5. После это появится краткая информация о выбранном материале корпуса.Если пользователь затрудняется в выборе, топо нажатию на кнопку «помощь», система подскажет необходимую информацию.

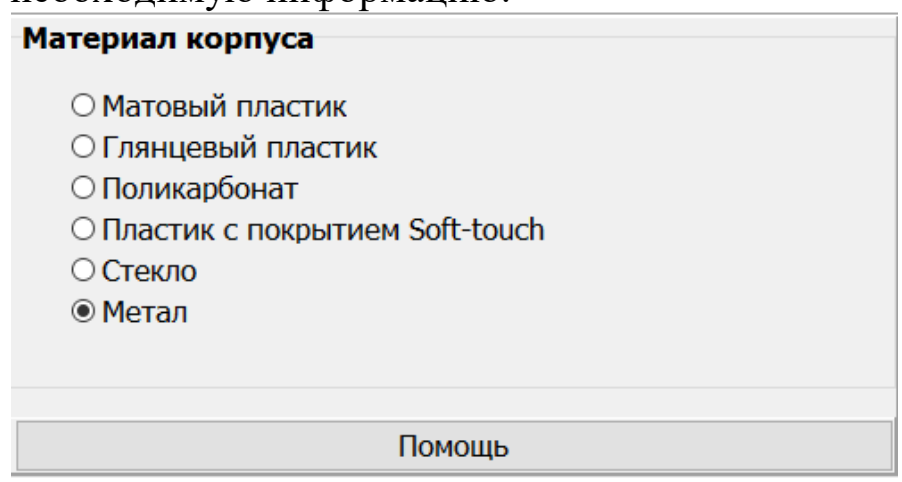

Рисунок5 -Поле выбора материала корпуса

После выбора всех характеристик критериев отбора смартфона необходимо нажать на кнопку «Выбор смартфона».

Далее системой будет произведен выбор самого оптимального смартфона, исходя из критериев отбора. Пример представлен на рисунке 6. Если пользователь захочет купить выбранный смартфон, то по нажатию на его название система перенаправит пользователя насайт интернет-магазина, где он сможет произвести покупку подобранного смартфона.

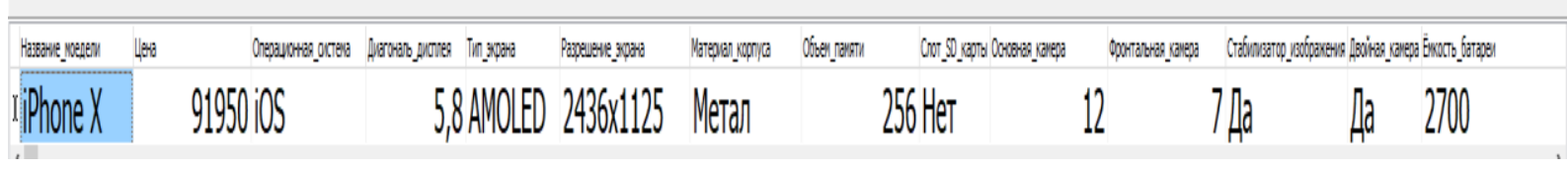

Рисунок 6-Пример выбранного смартфона

В конечном итоге система должна помочь потенциальным покупателям выбрать смартфон, который полностью соответствует их запросам.

$$
* * *
$$

1. Иноземцева С.А., Коновцова М.М., Рябова А.А., Черкалина Н.А., Баранов Р.Д.Информационные ресурсы и системы. Учебное пособие для студентов высших учебных заведений, обучающихся по направлениям подготовки 230400 "Информационные системы и технологии", 230700 "Прикладная информатика". - Пятигорск: РИА на КМВ, 2014.-160 с.

2. Интеллектуальные информационные системы и технологии [Электронный ресурс]: учебное пособие/ Ю.Ю. Громов [и др.].— Электрон.текстовые данные.— Тамбов: Тамбовский государственный технический университет, ЭБС АСВ, 2013.— 244 с.- Режим доступа: http://www.iprbookshop.ru/63850.html.— ЭБС «IPRbooks» 